\title{
Comparing Fruit and Tree Characteristics of Two Peaches and Their Nectarine Mutants
}

\author{
Ien-Chi Wen, K. E. Koch, and W. B. Sherman \\ Horticultural Sciences Department, University of Florida, Gainesville, FL 32611
}

Additional index words. Prunus persica, fruit size, fruit color, fruit sugars, fruit organic acids

\begin{abstract}
Two peach-to-nectarine mutants were compared with their peach progenitors to quantify physical and biochemical characters associated with this conversion. Both nectarine mutants showed pleiotropic effects that included smaller, rounder, and more-dense fruit with redder skin and altered sugar and organic acid composition relative to those found in their peach progenitors. In addition, one of the nectarine mutants exhibited a later bloom time, a less pronounced change in fruit size, a shorter fruit development period, and an associated capacity to develop red fall leaf color.
\end{abstract}

Nectarines arose as peach mutants, and their inheritance pattern is consistent with the glabrous skin characteristic controlled by a single recessive gene (Blake, 1932). Most aspects of nectarine trees, leaves, and flowers are indistinguishable from those of peach; however, peach researchers have noted differences between peaches and nectarines that extend beyond those of lack of pubescence alone. These differences include fruit size, shape, firmness, external color, aroma, flavor, and disease resistance, although such comparisons have not been quantified or analyzed statistically (Darwin, 1893; De Candolle, 1884; Downing, 1845; Hedrick, 1917; Hesse, 1975; Oberle and Nickolson, 1953). Hesse (1975), hypothesized that nectarine phenotypes such as those associated with ultrastructual differences in surface features (Fogle and Faust, 1975) represent a nonlethal deletion for surface pubescence that is closely linked to other genes affecting the fruit traits noted above. It is also possible that the nectarine phenotype may arise as an alteration in a single regulatory gene that controls the expression of other genes. This interpretation is consistent with genetic evidence and molecular characterization of the two known classes of glabrous mutations in Arabidopsis thaliana (L.) Heynh.[(glabrous (g11) and transparent testa glabrous (ttg])] (Koornneef, 1981; Koornneef et al., 1982).

A nectarine mutant was identified in 1988 on a limb of a 2-yearold 'TropicBeauty' peach tree in the Univ. of Florida breeding orchard. This mutant (designated TBN) showed evidence of having a higher chilling requirement and shorter fruit development period relative to its peach progenitor. Changes in such characteristics have not previously been observed in association with peachto-nectarine mutants. A second nectarine mutant was found on a limb of Fla. M3-1 peach in the same orchard 1 year later. This peach-to-nectarine mutants was designated M3-1N and appeared to be more typical of current commercial nectarines in fruit size and color, as summarized by Hesse (1975).

Our objective was to compare the two peach-to-nectarine mutants and contrast them to their peach progenitors. These isogenic materials have provided the first opportunity (beyond the single fruit observation by Oberle and Nicholson, 1953) to define the specific physical and biochemical attributes of peach-tonectarine mutants.

Received for publication 28 Feb. 1994. Accepted for publication 13 June 1994. Florida Agricultural Experiment Station Journal Series R03658. The cost of publishing this paper was defrayed in part by the payment of page charges. Under postal regulations, this paper thereforemust be hereby marked advertisement solely to indicate this fact.

\section{Materials and Methods}

Two peach genotypes ('TropicBeauty' and Fla. M3-1) and their nectarine mutants (TBN and M3-1N, respectively) were used in 1990,1991 , and 1992 to characterize the specific changes associated solely with the peach-to-nectarine mutants. Samples (fruits and leaves) were collected from nonmutant and mutant limbs on the mature original trees (ortets) and from 2- to 4-year-old, budded trees (clones $=$ ramets) of both peach genotypes and their nectarine mutants. Tree branches were thinned to about the same relative fruit : foliage ratio simulating commercial culture.

Twenty representative fruit judged ready for commercial harvest were harvested from each genotype in the ortets and ramets. Samples were pooled to compare fruit size, color, sugars, and organic acids. Ten fruit of each genotype were used for measuring specific gravity and fresh weight and dry weights. Fruit volume was measured by water displacement, and specific gravity was derived from fresh weight per volume. Dry weight was determined after drying fruit slices at $75 \mathrm{C}$ until weight reduction ceased. Twenty leaves of each genotype were collected from ortets and ramets, and samples of each were pooled to determine leaf color and weight. Each leaf was sampled from the sixth position behind the terminal leaf of a shoot that had ceased growth in fall.

Fruit shape for each genotype was determined from five categories ranging from 1 (a pronounced protuberance at the fruit apex) to 5 (round). The fruit development period was calculated as days from the date when $50 \%$ of the flower buds had opened to the date when fruit were judged ready for first commercial harvest. Foliation dates of the nectarine mutants and their peach progenitors were compared to those of selected cultivars to obtain estimates their chill unit requirements (Sherman et al., 1988).

Fruit color was measured with a optical sensor D25 Hunter-Lab standardized to a gray plate $(\mathrm{L}=51.37, \mathrm{a}=54.64, \mathrm{~b}=59.20)$. Lightness was measured on the ' $\mathrm{L}$ ' scale, which ranges from 0 for black to 100 for white. The degree of redness was measured on the 'a' scale, which is positive for red and negative for green. The degree of yellowness was measured on the 'b' scale, which is positive for yellow and negative for blue. All color measurements were made on unwashed, unbrushed fruit and leave, because no significant differences were found between brushed and unbrushed peaches (data not shown). Each fruit sample was measured at an equatorial line on sunlit and shaded sides. Every leaf was measured on both sides at its centermost point. The Minolta ' $a$ ' and ' $b$ ' values were used to compute hue angle [hue angle $\left.=\left(\tan ^{-1} \mathrm{~b} / \mathrm{a}\right)\right]$, a parameter correlated with the visual appearance of many foods (Clydesdale, 1978; Crassweller et al., 1991; Francis, 1952). The 
lower the value, the more red the hue is generally perceived.

Fruit soluble solids (soluble sugars and organic acids) were separated, identified, and quantified via high-performance liquide chromatography (HPLC). Fruit flesh was sampled from the sunlit and shaded sides of each fruit and stored at $-80 \mathrm{C}$ until analysis. Preliminary data (not shown) indicated that the sugar concentration from sunlit and shaded sides of fruit was similar; therefore, equal numbers of samples from each side were pooled for total fruit analyses. Frozen tissue ( 5 to $8 \mathrm{~g}$ ) was ground in liquid $\mathrm{N}$ and extracted in a mixture of 12 methanol : 5 chloroform : 3 water (M $: \mathrm{C}: \mathrm{W}$ ) (by volume) at an approximate ratio of 1 tissue : 4 media. Mannitol (0.1g) was added as an internal standard. Samples were heated in a $75 \mathrm{C}$ water bath for $20 \mathrm{~min}$. Chloroform and water were added to bring the final $\mathrm{M}: \mathrm{C}: \mathrm{W}$ ratio to $10: 6: 5$. Solutions were vortexed and centrifuged at $2600 \times \mathrm{g}$ for $5 \mathrm{~min}$ to remove lipids and pigments. The supernatant was pipetted into a clean tube, and the pellet re-extracted with $10 \mathrm{ml}$ of the M : C : Wsolution. Supernatants were combined, vortexed after adding $3 \mathrm{ml}$ water, and centrifuged at $2600 \times g$ for $5 \mathrm{~min}$. The supernatant was pipetted into a clean tube and 1 to $2 \mathrm{~g}$ polyvinylpolypyrrolidone was added. The solution was vortexed and centrifuged at $2600 \times g$ for $5 \mathrm{~min}$. The PVPP pellet was reextracted with $2 \mathrm{ml} \mathrm{H}_{2} \mathrm{O}$. Pooled fractions were evaporated to dryness and resuspended in a known volume of water. A 2-ml aliquot was passed through a $0.45 \mathrm{~mm}$ syringe filter before being injected into an HPLC system (Bio-Rad, Richmond, Calif.). Water flowing at $0.6 \mathrm{ml} \cdot \mathrm{min}^{-1}$ was used for the mobile phase. A Ca form cation-exchange column (HPX-87C; BioRad) was used at $85 \mathrm{C}$, and eluted peaks were detected with a refractometer (BioRad). Sugars were quantified using known standards. Tissue sugar concentrations were diluted using fresh weight values from each sample to approximate fluid volume (Dickson, 1979; Koch and Avigne, 1990; Lowell et al., 1989). Organic acids were also identified and quantified by HPLC. Procedures were as described above, except that $0.01 \mathrm{~g}$ oxalic acid was used as an internal standard for HPLC analyses. A column (HPX-87H; Bio$\mathrm{Rad}$ ) was used at $65 \mathrm{C}$ and the mobile phase was changed to 0.01 $\mathbf{N}$ sulfuric acid at a flow rate of $0.4 \mathrm{ml} \cdot \mathrm{min}^{-1}$. Individual organic acids were quantified using an ultraviolet monitor at $210 \mathrm{~nm}$ (Campbell and Koch, 1989).
Fruit transpiration rates were measured in the field using intact, attached fruit $\approx 70$ to 75 days after pollination. At this time, 'TropicBeauty' and TBN fruit were $-6.0 \mathrm{~cm}$ in diameter and those of Fla. M3-1 and M3-1N were $-4.8 \mathrm{~cm}$. A dew-point hygrometer (1100 DP) General Eastern, was used to measure transpiration after fruit were equilibrated for 10 to $20 \mathrm{~min}$ in a 0.5 -liter cuvette supplied with outside air moving at 0.5 liters $\cdot \mathrm{min}^{-1}$. During measurements, field air temperatures ranged from 28 to $37 \mathrm{C}$ and those inside the chambers ranged from 31 to $42 \mathrm{C}$. Field air humidity ranged from $75 \%$ to $80 \%$.

The least squares means statistical analyses was used for comparing unbalanced data.

\section{Results}

Fruit shape of the two peach genotypes and their nectarine mutants were contrasted (data not shown) and TBN and M3-1N nectarines were rounder. Peach-to-nectarine mutant fruit received mean ratings of 5 for maximal roundness on a 0 to 5 scale for shape. However, 'TropicBeauty' and Fla. M3-1 were rated 4 and 2, respectively.

Physical parameters of fruit from both peach genotypes and their peach-to-nectarine mutants are compared in Table 1. TBN fruit fresh weight was $78 \%$ as heavy as its progenitor, whereas M3$1 \mathrm{~N}$ nectarines weighed $-50 \%$ as much as those of the peach from which it originated. No difference was found between fresh weight of peach pits and those of their respective nectarine mutants. Dry weights were also lower in nectarine mutants than in their peach progenitors (Table 1), a result indicating that the smaller size was not due solely to less water in the nectarines. However, the total smaller nectarine size was associated with a disproportionate decrease in fresh weight relative to dry weight, and nectarine specific gravity was greater than that of their peach progenitors. No differences were observed in fruit transpiration rates when each peach genotype was compared to its nectarine mutant, however, M3- 1 and M3- $1 \mathrm{~N}$ seemed to have almost double the transpiration rates of 'TropicBeauty' and TBN.

Surface color was contrasted for peaches and their nectarine mutants on the sunlit and shaded sides of the fruit. In general, ' $L$ ',

Table 1. Physical fruit characteristics of two peach genotypes ('TropicBeauty' and Fla. M3- 1) and their peach-to-nectarine mutants (TBN and M3- 1N).

\begin{tabular}{|c|c|c|c|c|c|c|}
\hline & TropicBeauty & TBN & Significance & Fla. M3-1 & M3-1N & Significance \\
\hline Fresh weight, fruit" ${ }^{2}(\mathrm{~g})$ & 138 & 108 & $* *$ & 90 & 44 & $* *$ \\
\hline Fresh weight, pits (g) & 5.8 & 5.8 & NS & 4.8 & 5.3 & NS \\
\hline Dry weight, fruit (g) & 19.3 & 14.8 & $* *$ & 12.7 & 10.3 & $* *$ \\
\hline Specific gravity & 1.0 & 1.1 & $* *$ & 1.0 & 1.2 & $* *$ \\
\hline Transpiration $\left(\mathrm{m} / \mathrm{H}_{2} \mathrm{O} / \mathrm{cm}^{2} / \mathrm{h}\right)$ & 8.4 & 10.4 & NS & 16.4 & 20.5 & NS \\
\hline \multicolumn{7}{|l|}{ Color sun side ${ }^{x}$} \\
\hline $\mathrm{L}$ & 41.3 & 40.5 & NS & 44.6 & 39.5 & $* *$ \\
\hline a & 28.6 & 31.5 & $* *$ & 36.7 & 31.4 & $* *$ \\
\hline $\mathrm{b}$ & 22.7 & 20.8 & NS & 28.2 & 19.4 & $* *$ \\
\hline Hue & 37.3 & 32.5 & $* *$ & 37.0 & 31.3 & $* *$ \\
\hline \multicolumn{7}{|l|}{ Color shade side $^{x}$} \\
\hline $\mathrm{L}$ & 62.1 & 58.1 & $* *$ & 62 & 64.0 & NS \\
\hline a & 19.7 & 23.9 & $* *$ & 27.4 & 21.0 & $* *$ \\
\hline b & 44.7 & 38.6 & $* *$ & 45.1 & 43.2 & NS \\
\hline Hue angle & 58.0 & 58.0 & NS & 58.7 & 58.7 & NS \\
\hline
\end{tabular}

${ }^{2}$ Average of 3 years data of 20 samples per year.

"The L, a, b, and Hue angles represent 2 years data of 20 samples per year.

Ns,**Nonsignificant or significant at $P=0.01$. 
Table 2. Physical tree characteristics of two peach genotypes ('TropicBeauty' and Fla. M3-1) and their peach-to-nectarine mutants (TBN and M3-1).

\begin{tabular}{|c|c|c|c|c|c|c|}
\hline & TropicBeauty & TBN & Significance & Fla. M3-1 & M3-1N & Significance \\
\hline Leaf area $(\mathrm{cm})^{2}$ & 35.7 & 33.8 & NS & 33.4 & 34.9 & NS \\
\hline Leaf fresh weight (g) & 0.80 & 0.72 & NS & 0.56 & 0.57 & NS \\
\hline Estimated chill units & 150 & 250 & $* *$ & 241 & 258 & NS \\
\hline \multicolumn{5}{|c|}{ Fruit development period } & 81 & NS \\
\hline \multicolumn{7}{|l|}{ Fall leaf color } \\
\hline $\mathrm{L}$ & 53.5 & 39.3 & $* *$ & 84.4 & 84.7 & NS \\
\hline a & 7.0 & 6.8 & NS & 1.4 & -0.3 & NS \\
\hline b & 33.7 & 14.7 & $* *$ & 3.1 & 3.6 & NS \\
\hline Hue angle & 78.8 & 65.7 & $* *$ & 74.5 & $66 . ?$ & NS \\
\hline
\end{tabular}

${ }^{\bar{z}}$ Average of 3 years of observations.

'a', and ' $b$ ' values and hue angle were consistent with the range reported by Robertson et al. ( 1992) in various maturity grades of low-chill peaches.

Overall ' $L$ ' value on the sunlit side of the fruit did not differ detectably between 'TropicBeauty' and TBN; however, sunlit sides of M3-1N fruit were darker (lower ' $L$ ' value) than those of Fla. M3-1. The 'a' values indicated that sunlit sides of TBN fruit were generally more red than those of 'TropicBeauty' peaches; whereas, sunlit sides of Fla. M3-1 were generally more red than its nectarine mutant. The peaches frequently had higher mean ' $b$ ' values on their sunlit sides than their nectarine mutants, a result indicating that the peaches were often more yellow. In general, Hue angle values were smaller for nectarines than peaches; thus, nectarines would have been perceived as being redder.

The color values from the shaded sides of fruit also showed significant contrasts which varied with genotype. The shaded sides of TBN fruit were darker (lower ' $L$ ' value) than those of its peach progenitor, although no trend between shaded sides of fruit from Fla. M3- 1 and Fla. M3- 1N was observed. The shaded side 'a' value for 'TropicBeauty' was lower than for TBN; however, that of Fla. M3- 1 peach was higher than its nectarine mutant (consistent with observations for the sunlit side of peaches and their mutants). Both peach genotypes were more yellow (higher ' $b$ ' values) on the shaded side of their nectarine mutants. Values for perceived redness (hue angle) on the shaded sides of peaches and nectarines showed no significant differences.

In contrasting the shaded vs. sunlit sides of peaches and nectarines, the ' $a$ ' values were higher on the sunlit side, indicating fruit were more red. In addition, 'L', 'b', and hue angle values were lower on the sunlit sides of fruit showing darker, less yellow, and more perceived redness, respectively.

The broad pleiotropic nature of the peach-to-nectarine mutation also extended to characteristic beyond those of fruit. Although there were no significant differences in leaf size or fresh weight (Table 2), other alterations were evident. TBN trees bloomed 7 to 14 days later than 'TropicBeauty' peaches (indicating a higher chilling requirement); however, fruit ripened 3 to 7 days earlier. In contrast, Fla. M3-1 and M3-IN generally bloomed and ripened at the same time. The average fruit development period of TBN nectarine was 16 days less than that of the isogenic 'TropicBeauty' peach progenitor in all 3 years studied. Furthermore, senescing leaves of TBN were significantly darker (lower ' $L$ ' value), less yellow (lower ' $b$ '), and had a greater degree of perceived redness (lower hue angle) than those of its 'TropicBeauty' peach progenitor. Visual observation indicated that TBN, but not 'TropicBeauty', had red anthocyanin in fall leaves. No differences were evident in the ' $L$ ', ' $a$ ', ' $b$ ', or hue values in senescing leaves of M3-1N nectarines compared to those of Fla. M3-1 peaches.

Mean content of sucrose, glucose, fructose, sorbitol, and total sugars and "Brix were equal or higher in nectarines when compared to fruit of their peach progenitors (Table 3). Sucrose predominated in peaches and nectarines, accounting for $75 \%$ to $80 \%$ of the total sugar. Glucose and fructose accounted for $\approx 7 \%$ to $15 \%$ of the total sugar, and mean levels of both were slightly greater in nectarines than in fruit of their peach progenitors. Sorbitol was a minor component in peaches and nectarines, accounting for $\approx 1 \%$ to $7 \%$ of the total sugar, and the only difference was observed between M3-1 (1.2) and M3-1N (7.4).

The mean total sugar content of 'TropicBeauty', TBN, Fla. M31 , and M3-1N fruit were 14.4, 10.7, 9.0, and $5.9 \mathrm{~g} /$ fruit, respectively (data were calculated from fresh weight minus pits in Table 1 and total sugars in Table 3). Thus, TBN and M3-1N had 74\% and $66 \%$ as much total sugar per fruit as their respective peach progenitors.

Organic acid analysis of fruit from peaches and their nectarine mutants showed citric and malic acids predominated, accounting for $63 \%$ to $85 \%$ of the total, with lesser levels of succinic, quinic, and acetic acids present. However, relative amounts of these organic acids were altered in both nectarine mutants, and balance between types of acids shifted in different directions depending on which of the two peach-to-nectarine mutants was considered. The TBN nectarine mutant was accompanied by a significant overall increase in fruit citric, quinic, and succinic acids relative to those of its 'TropicBeauty' progenitor. Concomitant decreases were also observed in malic and acetic acids. The substantially lower malic acid content in TBN nectarines minimized any changes in total organic acid levels in these mutant fruit. In contrast, only malate and citrate levels were elevated in M3-1N nectarines vs. M3-1 peaches, and this occurred to such an extent that total organic acid levels in these fruit were higher than those of its isogenic peach.

Overall, M3-IN nectarines had higher sugar and organic acid concentrations than its peach progenitor, whereas these traits did not differ significantly between fruit of the other peach and nectarine pair. A rise in "Brix accompanied both peach-to-nectarine mutants, however.

\section{Discussion}

The present comparison of two peach-to-nectarine mutants and their peach progenitors has quantified several features that generally accompany mutation to the glabrous phenotype and the extent of variation. Fresh weight was consistently reduced in TBN and M3-1N nectarines, which weighed $78 \%$ and $50 \%$ as much as fruit 
Table 3. Sugar and organic acid composition of fruit from two peach genotypes ('TropicBeauty' and Fla. M3- 1) and their peachto-nectarine mutants (TBN and M3-1N). Data are based on an average of either 2 or 3 years with 20 samples per year.

\begin{tabular}{|c|c|c|c|c|c|c|}
\hline & TropicBeauty & TBN & Significance & Fla. M3-1 & M3-1N & Significance \\
\hline \multicolumn{7}{|c|}{$\begin{array}{l}\text { Sugars } \\
\left(\mathrm{mg} \cdot \mathrm{g}^{-1} \text { fresh weight }\right)\end{array}$} \\
\hline Sucrose & 82.5 & 80.8 & NS & 76.2 & 100.7 & $* *$ \\
\hline Glucose & 6.9 & 9.1 & $* *$ & 10.4 & 11.6 & NS \\
\hline Fructose & 10.8 & 12.1 & $* *$ & 12.3 & 14.8 & * \\
\hline Sorbitol & 4.7 & 4.5 & NS & 1.2 & 7.4 & $* *$ \\
\hline Total & 104.7 & 106.5 & NS & 100.1 & 134.5 & $* *$ \\
\hline${ }^{\circ}$ Brix & 11.4 & 12.9 & $* *$ & 10.7 & 14.9 & $* *$ \\
\hline \multicolumn{7}{|c|}{$\begin{array}{l}\text { Organic acids } \\
\left(\mathrm{mg} \cdot \mathrm{g}^{-1} \text { fresh weight }\right)\end{array}$} \\
\hline Citric & 7.2 & 8.3 & $*$ & 5.3 & 8.9 & * \\
\hline Malic & 10.8 & 6.1 & $* *$ & 5.2 & 9.0 & ** \\
\hline Quinic & 0.0 & 0.2 & $*$ & 2.7 & 1.9 & NS \\
\hline Succinic & 3.1 & 4.8 & $* *$ & 2.2 & 2.9 & NS \\
\hline Acetic & 0.4 & 0.2 & $*$ & 1.3 & 0.8 & NS \\
\hline Total & 21.6 & 19.7 & NS & 16.7 & 23.4 & $* *$ \\
\hline
\end{tabular}

of their peach progenitors, respectively (Table 1). The degree of weight reduction accompanying the M3- $1 \mathrm{~N}$ mutant was similar to the previous, single-fruit observation by Oberle and Nicholson (1953), who reported that a nectarine from a bud sport was $45 \%$ as heavy as its peach progenitor. However, the less marked weight reduction in TBN nectarines indicated that the extent of this trait varied with the specific peach-to-nectarine mutant. Nectarines that are epidermal $\left(\mathrm{L}_{1}\right)$ mutants over peach flesh $\left(\mathrm{L}_{2}\right)$ have been identified (Donini and Rosselli, 1977). These L, nectarine mutants keep the progenitor peach size and texture and do not breed as nectarines (germ cells arise from $\mathrm{L}_{2}$ ). Thus, TBN could be an $\mathrm{L}_{1}$ glaborous mutant. The differences $m$ fruit weight did not seem to be related to pit weights or water content alone. Nectarines had a lower fresh weight : dry weight ratio and greater specific gravity. These data are consistent with the possibility that nectarines may have a reduced cell number, reduced intercellular space or both, relative to their peach progenitors. Differences in peach size among trees of the same cultivar are due to differences in cell count, with cell size having a minor effect (Bradley, 1959). Scorza et al. (1991) observed that large-fruited peach cultivars ('Loring' and 'Suncrest') had more cells (up to 3.7 times) than small-fruited cultivars ('Bailey' and 'Boone County'). Specific gravity data also suggest that TBN and M3-1N nectarines had less intercellular space than their peach progenitors. In peach, the extent of air space development is much less extensive than in apple, and it increases gradually during the entire growth period. The specific gravity of ripe peaches averaged 0.99 (Westwood, 1962). The reduced fruit size accompanying the peach-to-nectarine mutants described here may be due to cell number, intercellular space, or both.

Quantifying of differences in surface color in the isogenic peach-nectarine mutant pairs showed that glabrousness was often associated with darker, less yellow fruit and a greater degree of perceived redness (hue angle) on the sunlit sides (Table 1). Although the perceived redness (lesser hue angle) of both nectarines was significantly more pronounced than their peach progenitors on only the sunlit sides, ' $a$ ' values for redness differed on the sunlit and shaded sides. This value indicated that TBN nectarine was redder on the sunlit side than 'TropicBeauty' peach, however, the reverse was true for the Fla. M3-1 peach and M3- $1 \mathrm{~N}$ nectarine pair. The reason for this apparent inconsistency between the two peachto-nectarine mutants may be due to the greater number of visible sugar speckles on nectarines M3-1N mutant. Sugar speckles are yellowish blotches interrupting the red pigment, usually more numerous on the distal end of the fruit, and mostly associated by peach breeders with sweeter-fruited cultivars. These small spots of reduced pigment displace and reduce the overall redness from anthocyanin accumulation. Yearly and genotypic differences were present, but did not counter the overall differences and sun and shade effects noted above.

An interesting feature of TBN nectarine was the extent of its influence on nonfruit traits (Table 2). Fruit development period and leaf pigment during senescence were altered in TBN, but not in M3-IN. Senescing leaves of TBN nectarine were darker and redder than those of its peach progenitor. A similar red color in senescing leaves was previously associated with a short fruit development period in related germplasm (Sherman et al., 1972) and probably represents this same trait. Such effects were not noted in an earlier, single-fruit description of a nectarine mutant (Oberle and Nicholson, 1953).

Elevated sugar levels do not necessarily accompany a peach-tonectarine mutant. Although the Brix was consistently greater in both nectarine mutants examined here, these values are actually based on osmotically active solutes rather than on sugars per se. Detectable differences in concentration of total fruit sugars were not apparent with TBN, despite significantly higher glucose and fructose levels. These hexoses were a relatively minor component of the total sugars, however, so their influence was minimal, even when data were expressed as $\mathrm{mmol} \cdot \mathrm{g}^{-1}$ fresh weight (data not shown). In contrast, M3-1N had significantly higher levels of fruit sucrose, and this corresponded to visually greater amounts of surface sugar speckles on M3-1 N fruit. These and other aspects of the M3-1N mutant suggest that it is more similar than TBN to the undefined mutants (Fogle and Faust, 1975) that seem to have given rise to our currently cultivated nectarines.

The higher sugar concentration in M3-1N nectarines compared to Fla. M3-1 peaches may be due to the reduced size and/or increased specific gravity of the nectarine. Faust (1989) reported that sugar concentration in apples increased as fruit specific gravity increased. This association was not significant in the TBN and 'TropicBeauty' pair; however, the extent of reduced size accompanying TBN was considerably less than that in M3-1N.

The lack of significant differences in total sugar concentration between 'TropicBeauty' peaches and TBN nectarines may also be partially related to the associated reduced fruit development period. Fruit growth curves determined from measuring fruit diameter during the 1990 growing season (data not shown) indicated 
that stage 2 and stage 3 of TBN development were reduced by 1 week relative to those of 'TropicBeauty'. During the final phase of fruit development, particularly the last 2 weeks of maturation, sucrose concentration increases rapidly in peaches (Ishida et al., 1971; Moriguchi et al., 1990). In addition, previous observations of breeding material over many years have indicated that peaches and nectarines selected for short fruit development period have lower sugar levels than genotypes with long fruit development periods. However, it is unclear whether the relatively small change in fruit development period reported here ( -2 weeks) can have the same effect as the much greater differences within the range of other existing germplasm (up to 16 weeks).

Both nectarine mutants accumulated less total sugar per fruit than their peach progenitors, despite greater concentrations of sugars per unit fresh weight in the nectarines (calculated from fruit fresh weights and total sugar per gram of tissue). Reduced total sugar per fruit was, as expected, based on decreased total size (fresh weight) in TBN and 'TropicBeauty'. Total sugar and mean fresh weight in TBN nectarines were $74 \%$ and $78 \%$, respectively, of those in 'TropicBeauty' peaches. Decreased total fruit sugar content was not as great as reduced size observed with M3-1N nectarines mutant (total sugar and fresh weight per nectarine fruit were $56 \%$ and $66 \%$ respectively of those for Fla. M3-1).

The changes in organic acid composition that accompanied the peach-to-nectarine mutants studied here have no obvious explanation. Overall organic acid concentration is higher in $\mathrm{M} 3-1 \mathrm{~N}$ nectarines than in Fla. M3-1 or 'TropicBeauty' and TBN, possibly because the fruit expand the least and dilute the organic acids less. However, it is not obvious why fruit citrate levels should be significantly elevated with both of the peach-to-nectarine mutants. In addition, differences between the two nectarines is not readily explained by the shortened fruit development period associated with the TBN mutant. TBN nectarines have less malic acid and less mean total acids than 'Tropic Beauty' or M3-1N. Acid concentration in peach is usually low at the beginning of fruit development, reaches a maximum in middevelopment, then steadily declines as the fruit mature (Ishida et al., 1971). The low level of malic acid in TBN fruit may be due to an alteration in some aspect of its synthesis, metabolism, or vacuolar compartmentalization (Campbell and Koch, 1989; Yen, 1987). Citric acid and malic acid are respiratory intermediates. Malic acid is also produced via $\mathrm{CO}_{2}$ fixation by phosphoenolpyruvate carboxylase and plays an important role in $\mathrm{pH}$ balance (Bown, 1985). In contrast to observations of the TBN mutant, a lower malate : citrate ratio is often typical of the most acidic cultivars and acidic mutants of other fruit types (Campbell and Koch, 1989; Yen, 1987).

Previous attempts to characterize the nature of peach-to-nectarine mutants have been minimal, probably due to the difficulty in locating confirmed peach progenitors. An isogenic peach-nectarine comparison was reported by Oberle and Nicholson (1953); however, only one fruit was available. Fogle and Faust (1975) found three different types of nectarine surface structures among currently cultivatednectarines. Although the peach progenitors for these are not known, their research revealed that the relatively small differences in nectarine surfaces seem to be heritable. Additional information was provided by Okie and Prince (1982), who observed that 3 of 70 trees from a 'Pekin' peach $\times$ 'Durbin' nectarine cross had a dull, slightly rough surface, intermediate between that of nectarine and peach. We have, since the completion of this study, identified two apparently different nectarine mutants, both arising from the same peach genotype, thereby providing evidence to the distinct genetic nature for individual nectarine mutants.
This study indicates that the nectarine locus controls or is closely linked with many other loci for features in addition to glabrous fruit skin. These distinct characteristics of TBN may bring us closer to understanding the pleiotropic nature of peach-tonectarine mutants. However, six seedlings, fruiting in Spring 1994, of TBN hybridized with two nectarine genotypes are peach. 'TropicBeauty' progeny hybridized with nectarine have produced $>50$ fruiting seedlings, all of which are peach. Thus, TBN is likely homozygous for peach in breeding $\left(\mathrm{L}_{2}\right)$ and homozygous for nectarine in skin $\left(\mathrm{L}_{1}\right)$ and seems to be of no value for breeding large-sized, early ripening nectarine cultivars.

\section{Literature Cited}

Blake, M.A. 1932. The J.H. Hale as a parent in peach crosses. Proc. Amer. Soc. Hort. Sci. 29:131-136.

Bown, A.W. 1985. $\mathrm{CO}_{2}$ and intracellular pH. Plant Cell Environ. 8:459465.

Bradley, M.V. 1959. Mean cell size in the mesocarp of mature peaches of different sizes. Proc. Amer. Soc. Hort. Sci. 73:120-124.

Campbell, C.A. and K.E. Koch. 1989. Sugar/acid composition and development of sweet and tart carambola fruit. J. Amer. Soc. Hort. Sci. 114:455-457.

Clydesdale, F.M. 1978. Colorimetry-Methodology and applications. CRC Critical Rev. Food Sci. Nutr. 10:243-301.

Crassweller, R.M., H.L. Braun, T.A. Baugher, G M. Greene, and R.A. Hollendar. 1991. Color evaluations of Delicious strains. Fruit Var. J. 45(2):114-120.

Darwin, C. 1893. The variation of animals and plants under domestication. D. Appleton and Co., New York.

De Candolle, A. 1884. Origin of cultivated plants. Kegan Paul, Trench \& Co., London.

Dickson, R.E. 1979. Analytical procedures for the sequential extraction of ${ }^{14} \mathrm{C}$-labeled constituents from leaves, bark, and wood of cottonwood plants. Physiol. Plant. 45:480-488.

Donini, B. and G. Rosselli. 1977. The use of mutation breeding to obtain nectarines. Riv. Ortoflorofrutt. It. 61:175-181.

Downing, A.J. 1845. The fruits and fruit trees of America. Wiley \& Putnam., London.

Faust, M. 1989. Physiology of temperate zone fruit trees. Wiley, New York.

Fogle, H.W. and M. Faust. 1975. Ultrastructure of nectarine fruit surfaces. Proc. Amer. Soc. Hort. Sci. 100:434-439.

Francis, F.J. 1952. A method of measuring the skin color of apple. Proc. Amer. Soc. Hort. Sci. 60:213.

Hedrick, U. P. 1917. The peaches of New York. J. B. Lyon, Albany, N.Y. Hesse, C.O. 1975. Peaches, p. 306-320. In: J. Janick and J.N. Moore (eds.). Advances in fruit breeding. Purdue Univ. Press., West Lafayette, Ind.

Ishida, M., A. Inaba, and Y. Sobajima. 1971. Seasonal changes in the concentration of sugars and organic acids in peach fruits. Sci. Rpt. Kyoto Prefecture Univ. Agr. 23: 18-23.

Koch, K.E. and W.T. Avigne. 1990. Postphloem, nonvascular transfer in citrus. Plant Physiol. 93:1405-1416.

Koomneef, M. 1981. The complex syndrome of ttg mutants. Arab. Info. Serv. 18:45-51.

Koornneef, M., L.W.M. Dellaert, and J.H. van der Veen. 1982. EMS- and radiation-inducedmutation frequencies at individual loci in Arabidopsis thaliana (L.) Heynh. Mutation Res. 93:109-123.

Lowell, C.A., P.T. Tomlinson, and K E. Koch. 1989. Sucrose-metabolizing enzymes in transport tissues and adjacent sink structures in developing citrus fruit. Plant Physiol. 90: 1394-1402.

Moriguchi, T., Y. Ishizawa, and T. Sanada. 1990. Differences in sugar composition in Prunus persica fruit and the classification by the principal component analysis. J. Jpn. Soc. Hort. Sci. 59:307-312.

Oberle, G.D. and J.O. Nickolson. 1953. Implications suggested by a peach to nectarine sport. Proc. Amer. Soc. Hort. Sci. 62:323-326.

Okie, W.R. and V.E. Prince. 1982. Surface features of a novel peach $\times$ 
nectarine hybrid. HortScience 17:66-67.

Robertson, J.A., F.I. Meredith, B.G. Lyon, G.W. Chapman, and W.B. Sherman. 1992. Ripening and cold storage changes in the quality characteristics of nonmelting clingstone peaches (Fla. 9-20C). J. Food Sci. 57(2):462465.

Scorza, R., L.G. May, B. Purnell, and B. Upchurch. 1991. Differences in number and area of mesocarp cells between small-and large-fruited peach cultivars. J. Amer. Soc. Hort. Sci. 116:861-864.

Sherman, W.B., P.M. Lyrene, N.F. Childers, F. G. Gmitter, and P.C.
Andersen. 1988. Low-chill peach and nectarine cultivars for trial in Florida. Proc. Florida State Hort. Soc. 101:241-244.

Sherman, W.B., R.H. Sharpe, and V.E. Prince. 1972. Two red leaf characters associated with early ripening peaches. HortScience 7:502-503.

Westwood, M.N. 1962. Seasonal changes in specific gravity and shape of apple, pear, and peach fruits. Proc. Amer. Soc. Hort. Sci. 80:90-96.

Yen, C.R. 1987. Assimilate partitioning and enzymes of organic acid metabolism in fruit of calamondin and low-acid grapefruit. $\mathrm{PhD}$ diss. Univ. of Florida., Gainesville. 